\title{
A NOTE ON CHEN'S BASIC EQUALITY FOR SUBMANIFOLDS IN A SASAKIAN SPACE FORM
}

\author{
MUKUT MANI TRIPATHI, JEONG-SIK KIM, and SEON-BU KIM
}

\author{
Received 28 January 2002
}

\begin{abstract}
It is proved that a Riemannian manifold $M$ isometrically immersed in a Sasakian space form $\tilde{M}(c)$ of constant $\varphi$-sectional curvature $c<1$, with the structure vector field $\xi$ tangent to $M$, satisfies Chen's basic equality if and only if it is a 3dimensional minimal invariant submanifold.
\end{abstract}

2000 Mathematics Subject Classification: 53C40, 53C25.

1. Introduction. Let $\tilde{M}$ be an $m$-dimensional almost contact manifold endowed with an almost contact structure $(\varphi, \xi, \eta)$, that is, $\varphi$ be a $(1,1)$-tensor field, $\xi$ be a vector field, and $\eta$ be a 1-form, such that $\varphi^{2}=-I+\eta \otimes \xi$ and $\eta(\xi)=$ 1. Then, $\varphi(\xi)=0, \eta \circ \varphi=0$, and $m$ is an odd positive integer. An almost contact structure is said to be normal, if in the product manifold $\tilde{M} \times \mathbb{R}$ the induced almost complex structure $J$ defined by $J(X, \lambda d / d t)=(\varphi X-\lambda \xi, \eta(X) d / d t)$ is integrable, where $X$ is tangent to $\tilde{M}, t$ is the coordinate of $\mathbb{R}$, and $\lambda$ is a smooth function on $\tilde{M} \times \mathbb{R}$. The condition for an almost contact structure to be normal is equivalent to the vanishing of the torsion tensor $[\varphi, \varphi]+2 d \eta \otimes \xi$, where $[\varphi, \varphi]$ is the Nijenhuis tensor of $\varphi$.

Let $g$ be a compatible Riemannian metric with the structure $(\varphi, \xi, \eta)$, that is, $g(\varphi X, \varphi Y)=g(X, Y)-\eta(X) \eta(Y)$ or equivalently, $g(X, \varphi Y)=-g(\varphi X, Y)$ and $g(X, \xi)=\eta(X)$ for all $X, Y \in T \tilde{M}$. Then, $\tilde{M}$ becomes an almost contact metric manifold equipped with the almost contact metric structure $(\varphi, \xi, \eta, g)$. Moreover, if $g(X, \varphi Y)=d \eta(X, Y)$, then $\tilde{M}$ is said to have a contact metric structure $(\varphi, \xi, \eta, g)$, and $\tilde{M}$ is called a contact metric manifold. A normal contact metric structure in $\tilde{M}$ is a Sasakian structure and $\tilde{M}$ is a Sasakian manifold. A necessary and sufficient condition for an almost contact metric structure to be a Sasakian structure is

$$
\left(\tilde{\nabla}_{X} \varphi\right) Y=g(X, Y) \xi-\eta(Y) X, \quad X, Y \in T \tilde{M}
$$

where $\tilde{\nabla}$ is the Levi-Civita connection of the Riemannian metric $g$. The manifolds $\mathbb{R}^{2 n+1}$ and $S^{2 n+1}$ are equipped with standard Sasakian structures. The sectional curvature $\tilde{K}(X \wedge \varphi X)$ of a plane section spanned by a unit vector $X$ orthogonal to $\xi$ is called a $\varphi$-sectional curvature. If $\tilde{M}$ has a constant 
$\varphi$-sectional curvature $c$, then it is called a Sasakian space form and is denoted by $\tilde{M}(c)$. For more details, we refer to [2].

Let $M$ be an $n$-dimensional submanifold immersed in an almost contact metric manifold $\tilde{M}(\varphi, \xi, \eta, g)$. Also let $g$ denote the induced metric on $M$. We denote by $h$ the second fundamental form of $M$ and by $A_{N}$ the shape operator associated to any vector $N$ in the normal bundle $T^{\perp} M$. Then $g(h(X, Y), N)=$ $g\left(A_{N} X, Y\right)$ for all $X, Y \in T M$ and $N \in T^{\perp} M$. The mean curvature vector is given by $n H=\operatorname{trace}(h)$, and the submanifold $M$ is minimal if $H=0$.

For a vector field $X$ in $M$, we put $\varphi X=P X+F X$, where $P X \in T M$ and $F X \in$ $T^{\perp} M$. Thus, $P$ is an endomorphism of the tangent bundle of $M$ and satisfies $g(X, P Y)=-g(P X, Y)$ for all $X, Y \in T M$. From now on, let the structure vector field $\xi$ be tangent to $M$. Then we write the orthogonal direct decomposition $T M=\mathscr{D} \oplus\{\xi\}$. Let $\left\{e_{1}, \ldots, e_{n}\right\}$ be an orthonormal basis of the tangent space $T_{p} M$. We can define the squared norm of $P$ by $\|P\|^{2}=\sum_{i, j=1}^{n} g\left(e_{i}, P e_{j}\right)^{2}$. For a plane section $\pi \subset T_{p} M$, we denote the functions $\alpha(\pi)$ and $\beta(\pi)$ of tangent space $T_{p} M$ into $[0,1]$ by $\alpha(\pi)=(g(X, P Y))^{2}$ and $\beta(\pi)=(\eta(X))^{2}+(\eta(Y))^{2}$, where $\pi$ is spanned by any orthonormal vectors $X$ and $Y$.

The scalar curvature $\tau$ at $p \in M$ is given by $\tau=\sum_{i<j} K\left(e_{i} \wedge e_{j}\right)$, where $K\left(e_{i} \wedge\right.$ $e_{j}$ ) is the sectional curvature of the plane section spanned by $e_{i}$ and $e_{j}$. The well-known Chen's invariant $\delta_{M}$ on $M$ is defined by

$$
\delta_{M}=\tau-\inf K
$$

where $(\inf K)(p)=\inf \left\{K(\pi) \mid \pi\right.$ is a plane section $\left.\subset T_{p} M\right\}$. For a submanifold $M$ in a real space form $\mathbb{R}^{m}(c)$, Chen [4] gave the following inequality:

$$
\delta_{M} \leq \frac{n^{2}(n-2)}{2(n-1)}\|H\|^{2}+\frac{1}{2}(n+1)(n-2) c
$$

He also established in [5] the similar basic inequalities for submanifolds in a complex space form. For an $n$-dimensional submanifold $M$ in a Sasakian space form $\tilde{M}(c)$ tangential to the structure vector field $\xi$ in [7], the authors established the following Chen's basic inequality.

THEOREM 1.1. Let $M$ be an $n$-dimensional $(n \geq 3)$ Riemannian manifold isometrically immersed in a Sasakian space form $\tilde{M}(c)$ of constant $\varphi$-sectional curvature $c<1$ with the structure vector field $\xi$ tangent to $M$. Then,

$$
\delta_{M} \leq \frac{n^{2}(n-2)}{2(n-1)}\|H\|^{2}+\frac{1}{8}\left\{n(n-3) c+3 n^{2}-n-8\right\}
$$

with equality holding if and only if $M$ admits a quasi-anti-invariant structure of rank $(n-2)$. 
For certain inequalities concerned with the invariant $\delta\left(n_{1}, \ldots, n_{k}\right)$, which is a generalization of $\delta_{M}$, we also refer to [6].

In this note, we prove the following obstruction to the Chen's basic equality.

THEOREM 1.2. Let $M$ be an $n$-dimensional Riemannian manifold isometrically immersed in an $m$-dimensional Sasakian space form $\tilde{M}(c)$ of a constant $\varphi$-sectional curvature $c<1$ with the structure vector field $\xi$ tangent to $M$. Then, $M$ satisfies the Chen's basic equality

$$
\delta_{M}=\frac{n^{2}(n-2)}{2(n-1)}\|H\|^{2}+\frac{1}{8}\left\{n(n-3) c+3 n^{2}-n-8\right\},
$$

if and only if $M$ is a 3-dimensional minimal invariant submanifold. Hence, Chen's basic equality (1.5) becomes

$$
\delta_{M}=2 .
$$

2. Proof of Theorem 1.2. First, we recall the following theorem [3].

THEOREM 2.1. Let $\tilde{M}$ be an $m$-dimensional Sasakian space form $\tilde{M}(c)$. Let $M$ be an $n$-dimensional $(n \geq 3)$ submanifold isometrically immersed in $\tilde{M}$ such that $\xi \in T M$. For each plane section $\pi \subset \mathscr{D}_{p}, p \in M$,

$$
\begin{aligned}
\tau-K(\pi) \leq & \frac{n^{2}(n-2)}{2(n-1)}\|H\|^{2}+\frac{1}{8}\left\{n(n-3) c+3 n^{2}-n-8\right\} \\
& +\frac{c-1}{8}\left\{3\|P\|^{2}-6 \alpha(\pi)\right\} .
\end{aligned}
$$

The equality in (2.1) holds at $p \in M$ if and only if there exist an orthonormal basis $\left\{e_{1}, \ldots, e_{n}\right\}$ of $T_{p} M$ and an orthonormal basis $\left\{e_{n+1}, \ldots, e_{m}\right\}$ of $T_{p}^{\perp} M$ such that (a) $e_{n}=\xi$, (b) $\pi=\operatorname{Span}\left\{e_{1}, e_{2}\right\}$, and (c) the shape operators $A_{r} \equiv A_{e_{r}}$, $r=n+1, \ldots, m$, take the following forms:

$$
\begin{aligned}
A_{n+1} & =\left(\begin{array}{ccccc}
h_{11}^{n+1} & 0 & 0 & \cdots & 0 \\
0 & -h_{11}^{n+1} & 0 & \cdots & 0 \\
0 & 0 & 0 & \cdots & 0 \\
\vdots & \vdots & \vdots & \ddots & \vdots \\
0 & 0 & 0 & \cdots & 0
\end{array}\right), \\
A_{r} & =\left(\begin{array}{ccccc}
h_{11}^{r} & h_{12}^{r} & 0 & \cdots & 0 \\
h_{12}^{r} & -h_{11}^{r} & 0 & \cdots & 0 \\
0 & 0 & 0 & \cdots & 0 \\
\vdots & \vdots & \vdots & \ddots & \vdots \\
0 & 0 & 0 & \cdots & 0
\end{array}\right), r=n+2, \ldots, m .
\end{aligned}
$$


A submanifold $M$ of an almost contact metric manifold $\tilde{M}$ with $\xi \in T M$ is called a semi-invariant submanifold [1] of $\tilde{M}$ if the distributions $\mathscr{D}^{1}=T M \cap$ $\varphi(T M)$ and $\mathscr{D}^{0}=T M \cap \varphi\left(T^{\perp} M\right)$ satisfy $T M=\mathscr{D}^{1} \oplus \mathscr{D}^{0} \oplus\{\xi\}$. In fact, the condition $T M=\mathscr{D}^{1} \oplus \mathscr{D}^{0} \oplus\{\xi\}$ implies that the endomorphism $P$ is an $f$-structure [9] on $M$ with a $\operatorname{rank}(P)=\operatorname{dim}\left(\mathscr{D}^{1}\right)$. A semi-invariant submanifold of an almost contact metric manifold becomes an invariant or an anti-invariant submanifold according as the anti-invariant distribution $\mathscr{D}^{0}$ is $\{0\}$ (i.e., $F=0$ ) or the invariant distribution $\mathscr{D}^{1}$ is $\{0\}$ (i.e., $P=0$ ) [1].

For each point $p \in M$, we put [3]

$$
\delta_{M}^{\mathscr{D}}(p)=\tau(p)-\left(\inf _{\mathscr{D}} K\right)(p)=\inf \left\{K(\pi) \mid \text { plane sections } \pi \subset \mathscr{D}_{p}\right\} .
$$

For $c<1$, we prove the following result.

THEOREM 2.2. Let $M$ be an $n$-dimensional $(n \geq 3)$ submanifold isometrically immersed in a Sasakian space form $\tilde{M}(c)$ such that the structure vector field $\xi$ is tangent to $M$. If $c<1$, then

$$
\delta_{M}^{\mathscr{D}} \leq \frac{n^{2}(n-2)}{2(n-1)}\|H\|^{2}+\frac{1}{8}\left\{n(n-3) c+3 n^{2}-n-8\right\} .
$$

The equality case in (2.4) holds if and only if $M$ is a 3-dimensional minimal invariant submanifold.

Proof. Since $c<1$, in order to estimate $\delta_{M}$, we minimize $\|P\|^{2}-2 \alpha(\pi)$ in (2.1). For an orthonormal basis $\left\{e_{1}, \ldots, e_{n}=\xi\right\}$ of $T_{p} M$ with $\pi=\operatorname{span}\left\{e_{1}, e_{2}\right\}$, we write

$$
\|P\|^{2}-2 \alpha(\pi)=\sum_{i, j=3}^{n} g\left(e_{i}, \varphi e_{j}\right)^{2}+2 \sum_{j=3}^{n}\left\{g\left(e_{1}, \varphi e_{j}\right)^{2}+g\left(e_{2}, \varphi e_{j}\right)^{2}\right\} .
$$

Thus, the minimum value of $\|P\|^{2}-2 \alpha(\pi)$ is 0 , provided that

$$
\operatorname{span}\left\{\varphi e_{j} \mid j=3, \ldots, n\right\}
$$

is orthogonal to the tangent space $T_{p} M$. Thus, we have (2.4) with equality case holding if and only if $M$ is a semi-invariant such that $\operatorname{rank}(P)=2$. This means that

$$
T M=\mathscr{D}^{1} \oplus \mathscr{D}^{0} \oplus\{\xi\}
$$

with the $\operatorname{dim}\left(\mathscr{D}^{1}\right)=2$. From (2.2), we see that $M$ is minimal. 
Next, from [8, Proposition 5.2], we have

$$
A_{F X} Y-A_{F Y} X=\eta(X) Y-\eta(Y) X, \quad X, Y \in \mathscr{D}^{0} \oplus\{\xi\} .
$$

For $X \in \mathscr{D}^{0}$ and using (2.8), we have

$$
\mathcal{g}(X, X)=-g\left(A_{F X} \xi, X\right),
$$

which in view of (2.2) becomes zero. Thus $\mathscr{D}^{0}=\{0\}$, and $M$ becomes invariant. This completes the proof.

From (1.2) and (2.3), it follows that $\delta_{M}^{\mathscr{D}}(p) \leq \delta_{M}(p)$. Hence in view of Theorem 2.2, we get the proof of Theorem 1.2.

REMARK 2.3. In Theorem 1.1, the phrase " $M$ admits a quasi-anti-invariant structure of $\operatorname{rank}(n-2)$ " is identical with the statement " $M$ is a semi-invariant submanifold with $\operatorname{rank}(P)=2$ or equivalently $\operatorname{dim}\left(\mathscr{D}^{1}\right)=2$, where $\mathscr{D}^{1}$ is the invariant distribution." Thus, nothing is stated here about the dimension of the anti-invariant distribution $\mathscr{D}^{0}$. But, in the proof of Theorem 2.2, we observe that $M$ becomes minimal and consequently invariant, which makes $\operatorname{dim}\left(\mathscr{D}^{0}\right)=0$ and $\operatorname{dim}(M)=3$.

Acknowledgment. This work was done while the first author was a Postdoctoral Researcher in the Brain Korea-21 Project at Chonnam National University, Korea.

\section{REFERENCES}

[1] A. Bejancu, Geometry of CR-Submanifolds, Mathematics and Its Applications (East European Series), vol. 23, D. Reidel Publishing, Dordrecht, 1986.

[2] D. E. Blair, Contact Manifolds in Riemannian Geometry, Lecture Notes in Mathematics, vol. 509, Springer-Verlag, Berlin, 1976.

[3] A. Carriazo, A contact version of B.-Y. Chen's inequality and its applications to slant immersions, Kyungpook Math. J. 39 (1999), no. 2, 465-476.

[4] B.-Y. Chen, Some pinching and classification theorems for minimal submanifolds, Arch. Math. (Basel) 60 (1993), no. 6, 568-578.

[5] _ A general inequality for submanifolds in complex-space-forms and its applications, Arch. Math. (Basel) 67 (1996), no. 6, 519-528.

[6] F. Defever, I. Mihai, and L. Verstraelen, B.-Y. Chen's inequalities for submanifolds of Sasakian space forms, Boll. Unione Mat. Ital. Sez. B Artic. Ric. Mat. (8) 4 (2001), no. 2, 521-529.

[7] Y. H. Kim and D.-S. Kim, A basic inequality for submanifolds in Sasakian space forms, Houston J. Math. 25 (1999), no. 2, 247-257.

[8] M. M. Tripathi, Almost semi-invariant submanifolds of trans-Sasakian manifolds, J. Indian Math. Soc. (N.S.) 62 (1996), no. 1-4, 225-245.

[9] K. Yano and M. Kon, Structures on Manifolds, Series in Pure Mathematics, vol. 3, World Scientific Publishing, Singapore, 1984. 
Mukut Mani Tripathi: Department of Mathematics and Astronomy, Lucknow University, Lucknow 226 007, India

E-mail address: mm_tripathi@hotmai1.com

Jeong-Sik Kim: Department of Mathematics Education, Sunchon National University, Sunchon 540-742, Korea

E-mail address: jskim01@hanmi r.com

Seon-Bu Kim: Department of Mathematics, Chonnam National University, Kwangju 500-757, Korea

E-mail address: sbk@chonnam.chonnam.ac.kr 


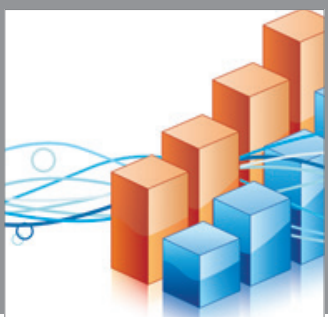

Advances in

Operations Research

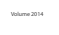

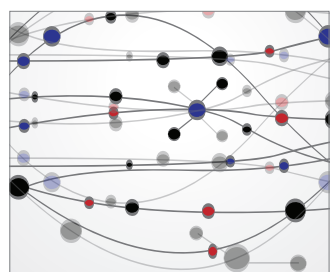

\section{The Scientific} World Journal
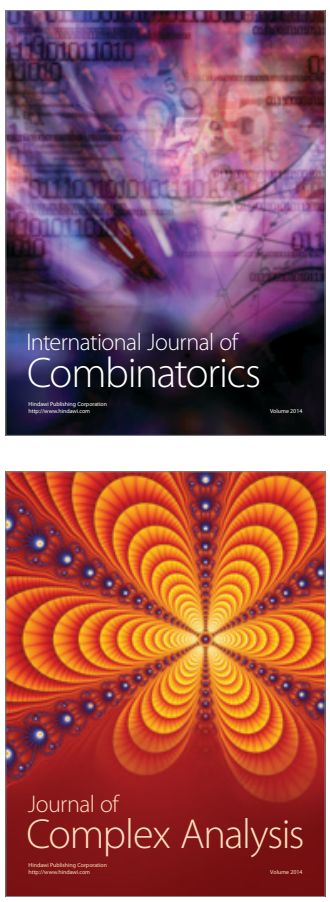

International Journal of

Mathematics and

Mathematical

Sciences
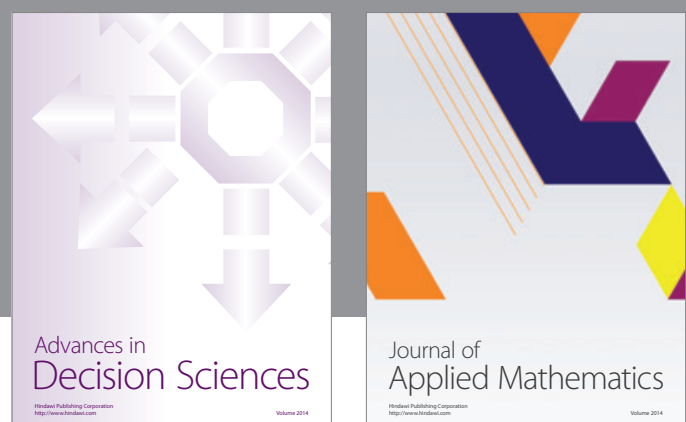

Journal of

Applied Mathematics
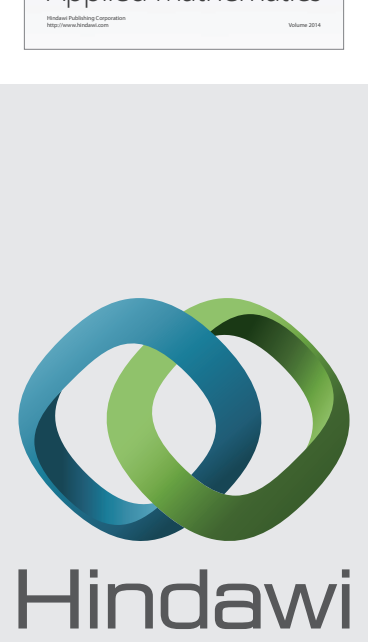

Submit your manuscripts at http://www.hindawi.com
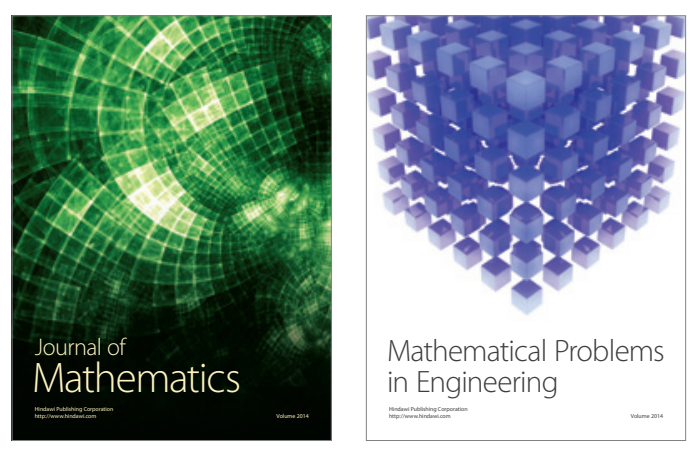

Mathematical Problems in Engineering
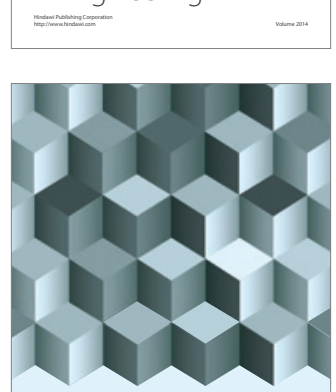

Journal of

Function Spaces
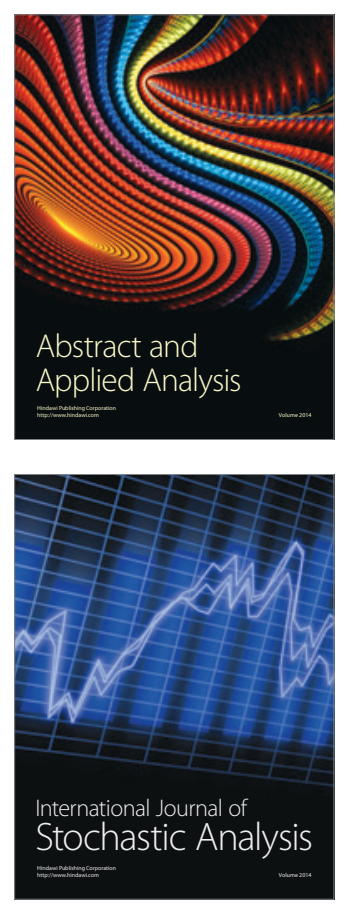

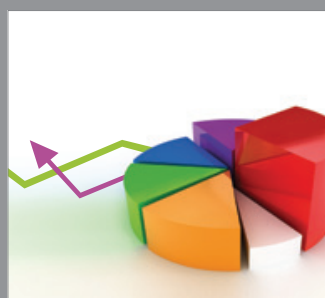

ournal of

Probability and Statistics

Promensencen
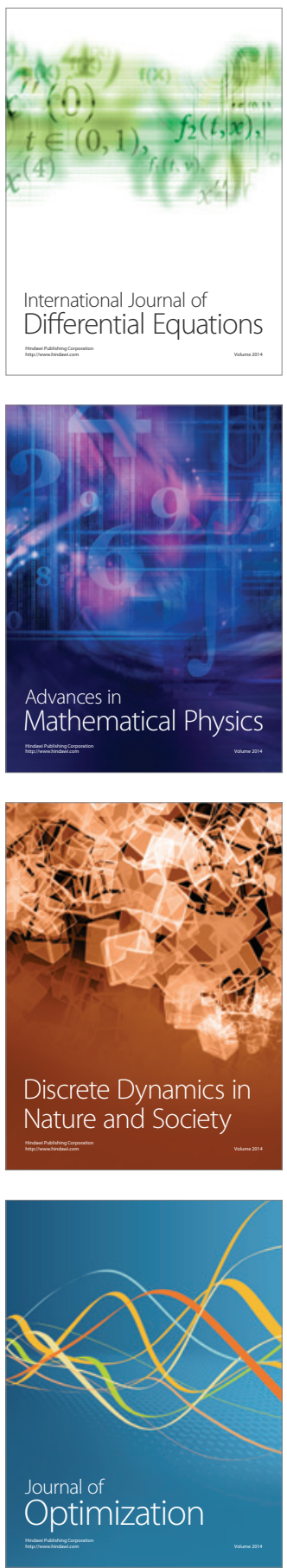